Research Paper

\title{
LncRNA-PVT1 indicates a poor prognosis and promotes angiogenesis via activating the HNF1B/EMT axis in glioma
}

\author{
Yongyan $\mathrm{Bi}^{1,2}$, Jie $\mathrm{Ji}^{3}$, Youxin Zhou ${ }^{1 凶}$ \\ 1. Department of Neurosurgery \& Brain and Nerve Research Laboratory, The First Affiliated Hospital of Soochow University, Suzhou, Jiangsu, China \\ 2. Department of Neurosurgery, Minhang Hospital, Fudan University, Minhang, Shanghai, China \\ 3. Department of Rehabilitation Medicine, Minhang Hospital, Fudan University, Minhang, Shanghai, China \\ $\triangle$ Corresponding author: You-Xin Zhou. zhouyouxin@suda.edu.cn. Department of Neurosurgery and Brian and Nerve Research Laboratory, The First \\ Affiliated Hospital of Soochow University, 899 Pinghai Street, Suzhou, Jiangsu, 215006, China \\ (C) The author(s). This is an open access article distributed under the terms of the Creative Commons Attribution License (https://creativecommons.org/licenses/by/4.0/). \\ See http:/ /ivyspring.com/terms for full terms and conditions.
}

Received: 2021.03.09; Accepted: 2021.07.12; Published: 2021.07.25

\begin{abstract}
Recent studies identified that long non-coding RNAs (IncRNAs) exhibited critical roles in tumor migration and invasion. However, the roles of IncRNAs in glioma remain unclear. The aim of this study was to uncover the underlying mechanisms of glioma progression and provide potential therapeutic targets for its treatment in clinic. Our microarray study showed that IncRNA-PVT1 was significantly upregulated in glioma tissues and played an important role in cell proliferation, migration, invasion and angiogenesis. Our data showed that the expression of IncRNA-PVT1 was increased obviously and associated with advanced tumor stage, metastasis, invasion ability, and poor prognosis in glioma patients. Up-regulation of IncRNA-PVT1 was observed to promote glioma cells proliferation, and invasion abilities in vitro as well as tumor growth in vivo by regulating miR-1207-3p expression. Online software (TargetScan, miRDB and miR TarBase) were used to predict the regulating mechanisms of IncRNA-PVTI, miR-1207-3p and HNF1B, which were validated by dual-luciferase reporter gene system. In vivo tumor-bearing mice models were established to validate the cellular results. Therefore, we suggested that IncRNA-PVT1/miR-1207-3p/HNF1B axis might play critical roles in glioma progression, indicating that IncRNA-PVT1/miR-1207-3p/HNFIB signaling axis may serve as novel molecular targets for glioma prevention and treatment.
\end{abstract}

Key words: lncRNA-PVT1, HNF1B, miR-1207-3p, glioma, EMT

\section{Introduction}

Human glioma that is derived from the neural ectoderm is the most common type of intracranial neoplasm, accounting for more than 50\% [1]. Although therapeutic techniques, such as surgery, radiotherapy, and chemotherapy for glioma have achieved great strides, the median survival of glioma patients is only 9-12 months [2-3]. The poor prognosis and high recurrence rate of glioma is largely due to the abundant blood flow, rapid growth, susceptibility of invasion to surrounding normal brain tissues and resistance to radiotherapy and chemotherapy [4]. The tumorigenesis of glioma is biologically complex, involving a series of pathophysiological changes, and expression changes of many genes and signaling pathways [5]. Thus far, there is no definite conclusion about the underlying molecular mechanisms that mediate the tumorigenesis of brain glioma. Therefore, it is necessary to uncover the pathogenesis of glioma and develop effective diagnostic and therapeutic targets.

Long non-coding RNAs (lncRNAs) belong to ncRNA with a transcription length of more than 200 nt (nucleotides) and lack of protein coding functions [6]. Recently, accumulated evidence showed that IncRNAs play crucial roles in cellular functions, such as cell proliferation, apoptosis, differentiation, 
metastasis, drug resistance [7-8]. Plasmacytoma variant translocation 1 (lnc-PVT1) is a lncRNA that has been found to serve an oncogenic role in a variety of malignant tumors [9]. Recent evidence further indicates that IncRNA-PVT1 exhibits aberrant expression in nonsmall-cell lung carcinoma [10], cervical carcinoma [11], colorectal carcinoma [12], and gastric carcinoma [13]. Located at 8q24, lncRNA-PVT1 has been identified as a candidate oncogene and highly expressed in multiple human neoplasms, which exerts regulatory functions in biological processes, such as proliferation, apoptosis, mobility, and invasion [14]. Moreover, IncRNA-PVT1 expression is significantly linked to patient survival in those with breast carcinoma, IncRNA-PVT1 has been shown to directly bind and stabilize the KLF5 proteins in breast cancer [15]. EZH2 forms a molecular complex with lncRNA-PVT1 to function as a repressive driver of p15 and p16 in gastric cancer [16]. LncRNA-PVT1 is also transcriptionally activated by FOXM1 in gastric cancer [17]. Furthermore, lncRNAPVT1 induces radioresistance by influencing cell apoptosis and DNA repair in NPC [18]. Furthermore, the specific role of lncRNA lncRNA-PVT1 in the pathogenesis of glioma has become a viewpoint, as there were quite a few studies on the roles of lncRNA-PVT1 in glioma [19-20]. However, the underlying lncRNA-miRNA-mRNA regulatory networks involved with lncRNA-PVT1 in glioma are currently lacking.

MicroRNA has been frequently implicated in various cellular processes, including cell proliferation and apoptosis [21-23]. Emerging evidences revealed that miR-30 family was related with tumor develepment. For example, Zhao et al. showed that miR-30-5p functioned as a tumor suppressor by regulating $\mathrm{Wnt} / \beta$-catenin-BCL9 axis [24]. Han et al. revealed that there is a feedback loop between miR-30a/c-5p and DNMT1 in the area of ovarian cancer cells resistance to cisplatin [25]. Gao et al. found that miR-30c-5p reduced invasion and EMT processes of gastric cancer via targeting MTA1 [26]. Some previous researches have indicated that miR-1207-3p was significantly upregulated, which might be potential cancer oncogene in different types of cancers, such as prostate cancer [27], gastric cancer [28], pancreatic cancer [29], and colorectal cancer [30]. Besides, miR-1207-3p has been found to exhibit a low expression and inhibit cell proliferation, suppress tumor growth, and block angiogenesis in medulloblastoma [31]. Nevertheless, the regulatory function of lncRNA-PVT1/miR-1207-3p axis in glioma cells needs to be further explored.

Herein, the purpose of the present study was to investigate the explicit role of lncRNA-PVT1 in the pathogenesis of glioma as well as the potential mechanism of lncRNA-PVT1/ miR-1207-3p/HNF1B axis in glioma. In the present study, we found that lncRNA-PVT1 was upregulated in glioma, and that it predicted poor survival in patients. We delved into the lncRNA-miRNA-mRNA networks by co-expressing lncRNA and mRNA with altered miRNA. Bioinformatics analysis was used to predict possible pathways in which these networks might be involved.

\section{Materials and Methods}

\section{Tissue samples collection}

In our current study, glioma patient tissues and matched adjacent-tumor controls were obtained from the Department of Neurosurgery at Minhang Hospital of Fudan University, between May 2015 and December 2019. The collected tissues were immediately snap-frozen in liquid nitrogen and stored at $-80^{\circ} \mathrm{C}$ before using. All the patients read and signed the informed consent forms and this study was approved by the Research Ethic Committee of the Fudan of University. Before surgery, none of the patients had received chemotherapy or radiation therapy.

\section{Cell culture and transfection}

Human glioma cell lines U87, SHG-44, U251, H4, human umbilical vein endothelia cells (HUVEC), human embryonic kidney 293T (HEK293T) cell line and normal cells (HEB) were purchased from the Cell Resource Center of Shanghai Institutes for Biological Sciences (Shanghai, China). Human microglia (HM, ATCC ${ }^{\circledR}$ CRL-3304 ${ }^{\mathrm{TM}}$ ) was purchased from the American Type Culture Collection (Manassas, VA). Glioma cells were cultivated in DMEM containing $10 \%$ fetal bovine serum (FBS; Sigma-Aldrich, St.Louis, $\mathrm{MO}, \mathrm{USA}$ ) in an incubator with constant temperature containing $5 \% \quad \mathrm{CO}_{2}$ at $37^{\circ} \mathrm{C}$. Glioma cells were transduced with lentiviral vector suppressing lncRNA-PVT1 (sh-lncRNA-PVT1), miR-1207-3p mimics, miR-1207-3p inhibitors and corresponding negative controls. All synthesis (sh-lncRNA-PVT1, miR-1207-3p mimics, miR-1207-3p inhibitors) are done by Shanghai IBSBIO. Cells were transfected with shRNA or miRNA via Lipofectamine 3000 reagents (Invitrogen, USA).

\section{RNA extraction and quantitative real-time PCR}

Total RNA was extracted from these included Glioma cells using TRIzol reagent based on the manufacturer's instructions [32]. The cDNA was synthesized using Reverse Transcription Kit (Toyobo, Japan). qPCR assay was conducted using SYBR Green Real-time PCR Mix (Toyobo, Japan) and determined 
in triplicate. The primer sequence of lncRNA-PVT1: 5'-GGGGAATAACGCTGGTGGAA-3', 5'-CCCATGG ACATCCAAGCTGT-3'. Data were normalized to GAPDH expression. The relative expression of target genes were analyzed by the $2-\Delta \Delta \mathrm{Ct}$ method.The PCR program was set as follows: $50^{\circ} \mathrm{C}$ for 2 min firstly, followed by $95^{\circ} \mathrm{C}$ for $10 \mathrm{~min}$, and 38 cycles of $95^{\circ} \mathrm{C}$ for $15 \mathrm{~s}$ and $60^{\circ} \mathrm{C}$ for $1 \mathrm{~min}$. U6 and GAPDH were served as internal reference.

\section{Microarray analysis}

Total RNA was extracted from 3 paired glioma tissues, Tissue samples preparation and microarray hybridization were performed according to the instructions. The raw data and array images were extracted with Agilent Feature Extraction (version11, Agilent, USA) software. Subsequent data processing was done using the GeneSpring GX v12.0 (Agilent). The microarray was provided by KangChen (Shanghai, China). The threshold cutoff was a fold change $\geq 2.0$ and a $P$ value $\leq 0.05$ for downregulated and upregulated lncRNAs.

\section{Western blot assay}

Total proteins from cells were extracted using RIPA lysis buffer (Thermo Fisher, USA) and protein concentrations were measured with BCA protein assay (Pierce, IL, USA). Totally $45 \mu \mathrm{g}$ of each protein was separated through 15\% SDS-PAGE gels and transferred onto PVDF membranes according to the instructions (Millipore, USA). After blocking with 5\% skimmed milk, the membranes were treated with primary antibodies at $4^{\circ} \mathrm{C}$ overnight. Then the corresponding secondary antibody was used for $1 \mathrm{~h}$ at room temperature. Protein bands were visualized by ECL (Thermo Fisher, USA). Soluble proteins were analyzed by immunoblotting with anti-HNF1B (07-2192; Sigma-Aldrich), anti-foxm1 (SAB3300002; Thermo Scientific), anti-Vimentin (ab8069; Abcam), anti-N-Cadherin (ab18203; Abcam), anti-p-ERK (mAb \#4370), anti-ERK (mAb \#4695), anti-p65 (mAb \#8242), anti-ZEB1 (mAb \#3396; Cell signaling Technology) and anti-GAPDH (G8795; Sigma).

\section{Immunohistochemistry staining and scoring}

The experimental methods are based on previous study. Specifically, staining intensity was scored manually by two independent experienced pathologists. For evaluation of IHC staining, a semiquantitative scoring criterion was used, in which both staining intensity and positive areas were recorded. Obtained as the intensity of HNF1B positive staining (negative $=0$, weak $=1$, moderate $=2$, or strong $=3$ scores) and the proportion of immunopositive cells of interest $(<10 \%=1,10 \%-50 \%=2,>50 \%$ and $<80 \%=3$, $>80 \%=4$ scores), was calculated. In the case of heterogeneous staining, we determined the percentage of different staining intensities individually in each area and calculated the total sum (3). The following primary antibodies were used: anti-HNF1B (1:200 dilutions, Cell Signaling, Boston, USA).

\section{Cell proliferation assay}

The cell proliferation viability was assessed by MTT assay (IBSBIO, China). Cells were seeded on 96-well plates in triplicates at the density of 1000-2000 cells/100 ml. Thereafter, $20 \mu \mathrm{l}$ of MTT solution was added and incubated at $37^{\circ} \mathrm{C}$ for $5 \mathrm{~h}$. The reaction liquid was abandoned, then $200 \mu \mathrm{l}$ of DMSO was added. Finally, the optical density 470 (OD 470) absorbance values were obtained in triplicate and replicated 6 times.

\section{Cell cytoplasm/nucleus fraction isolation}

To prepare cytoplasmic and nuclear extracts from glioma cells, NE-PER Nuclear and Cytoplasmic Extraction Reagents (Thermo Scientific, Waltham, MA, USA) was used. RT-qPCR was employed to analyze the levels of nuclear control transcript, cytoplasmic control transcript and lncRNA-PVT1.

\section{Transwell assay}

Cell invasion ability was carried out by Transwell invasion assay. Briefly, transfected cells were seeded into 24-well upper chamber (Corning, CA USA) pre-coated with Matrigel (Sigma, MO, USA). The lower chamber was filled with DMEM medium containing 10\% FBS. After culturing for $24 \mathrm{~h}$, the invade cells were stained with $0.1 \%$ crystal violet (Sigma) for $10 \mathrm{~min}$. The number of invaded cells was counted under a light microscope (Nikon, Japan).

\section{Luciferase reporter assay}

lncRNA-PVT1 and HNF1B 3'UTR Wt and mutant DNAs were amplified by PCR, then separately inserted into pmiR-GLO vector (Promega) to construct a Wt and Mut type of lncRNA-PVT1 and HNF1B plasmids. Cells were transfected with Wt or Mut reporter plasmid and miR-1207-3p mimics by lipofectamine 3000 reagent (Invitrogen). After $48 \mathrm{~h}$ of transfection, the luciferase activity was measured by Dual-luciferase reporter assay system (Promega, CA, USA).

\section{Tumor xenograft implantation in nude mice}

All BALB/c nude mice were purchased from Shanghai Laboratory Animal Center (Shanghai, China). Before the experiments, the mice were acclimatized to the new environment for one week. Mice were randomized into different groups with approximately equivalent numbers before tumor cell inoculation. To determine the effects of lncRNA-PVT1 
on tumor formation in vivo, glioma cells stably transfected with sh-lncRNA-PVT1 or sh-NC $\left(2.0 \times 10^{6}\right.$ cells/site) were injected subcutaneously into 4-6 weeks old female mice. The developing tumors were observed during the following 7 weeks, after which the mice were sacrificed. The weights of each tumor were also documented. Tumor volume was calculated as $\mathrm{V}=$ length $\times\left(\right.$ width $\left.^{2}\right) / 2$. The protocol was approved by the Institutional Animal Care and Use Committee of Fudan University.

\section{Gene Set Enrichment Analysis (GSEA)}

GSEA (http://software.broadinstitute.org/gsea /msigdb/index.jsp) software programs were used to analyze The Cancer Genome Atlas (TCGA) dataset. To interpret the function of regulated genes, GSEA (version 2.2.0) was performed using the 50 cancer hallmark gene sets and a gene log2-fold change. To identify the pathways that are correlated with LncRNA-PVT1 expression in tumor samples, we performed a similar GSEA for each cancer type in TCGA dataset. In this analysis, GSEA was performed on the ranked list based on the Spearmen's correlation coefficient with LncRNA-PVT1 expression.

\section{Statistical analysis}

In the present study, all of the statistical analysis was employed using SPSS 17.0 software package (SPSS Inc. Chicago, USA), GraphPad Prism 8.0 software (GraphPad, USA), and Microsoft office 2019 EXCEL (USA). $p<0.05$ was considered statistically significant. Pearson $\chi^{2}$ or Fisher's exact test was performed to find out the associations between the indicators expression and clinicopathological parameters in our included glioma patients. Linear regression analysis was explored to analyze the correlation of lncRNA-PVT1 with other indicators. Student's t-test (two-tailed) and Wilcoxon or Welch's T-test were used to compare the significance differences of the paired and unpaired continuous variables between groups, presented as mean \pm standard deviation (s.d.) or median (quartile).

\section{Results}

\section{Microarray analysis demonstrates significant alterations in IncRNA expression patterns in human glioma}

Differentially expressed lncRNAs (fold change $\geq$ 1.5 and $p<0.05$ ) were observed in Figure 1A, there are 327 lncRNAs exhibited high expression levels and 559 lncRNAs exhibited low expression levels. Among the dysregulated IncRNA transcripts, the mostly upregulated lncRNAs were lncRNA-PVT1 and lnc-004831, of which IncRNA-PVT1 was the highest $\left(\log _{2} \mathrm{FC}=4.32\right)$. In an attempt to further explore the function of lncRNA-PVT1 in a lncRNA-dependent manner, we first focused on 3 microarray data, GSE29384, GSE7181 and GSE23806 through searching GEO datasets (Figure 1B). As shown in Figure 1C, 327 genes were upregulated and 559 genes were downregulated, Scatter plot of gene expression in glioma compared with the control group. Then, gene ontology analysis (GO) was used to explore the roles of these differentially expressed lncRNAs. Results showed the majority of lncRNAs were assessed to be related to cancer progression (molecular function, cellular components and biological processes) (Figure 1D, E). Subsequently, GSEA results from microarray database showed that high lncRNA-PVT1 expression was associated with wnt pathway, cell adhesion and disease free survival of patients with glioma (Figure 1F, G). Therefore, we suggested that IncRNA-PVT1 might play critical roles in glioma progression.

\section{High IncRNA-PVT 1 expression in glioma cells and tissues}

To enrich the dysregulated lncRNAs-mRNAs and their correlations, the tumor development data were collected from TCGA Pan-Cancer database. The results illustrated that the expression of lncRNAPVT1 was pronouncedly increased in glioma tumor tissue compared to normal tissues. In most other solid tumors, such as breast invasive carcinoma, cervical squamous cell carcinoma and endocervical adenocarcinoma and cholangio carcinoma, IncRNA-PVT1 showed the opposite expression level (Figure 2A, B) $(p<0.001)$. Further, the expression level of lncRNA-PVT1 in glioma gradually increased with the increase of clinical stage, and the difference was significant $(p<0.05)$ (Figure 2C).

To assess the roles lncRNA-PVT1 in glioma, qRT-PCR showed that IncRNA-PVT1 was significantly upregulated in glioma tissue compared to adjacent non-tumor tissues (Figure 2D). High lncRNA-PVT1 expression was increased in glioma patients with advanced TNM stage and metastasis (Figure 2E, F). Furthermore, we analyzed the relationship between lncRNA-PVT1 expression and clinicopathologic features. Results showed that high lncRNA-PVT1 expression was associated with advanced tumor stage, metastasis, OS and DFS (Figure 2G, H). These data indicated that lncRNAPVT1 might be involved in glioma metastasis.

To further determine the underlying mechanisms of lncRNA-PVT1 on glioma progression, loss-of-function assays were used. The expression of lncRNA-PVT1 was significantly reduced in U251 and U87 cells transfected with sh-lncRNA-PVT1 (Figure 3A-C). MTT assays showed that IncRNA-PVT1 suppression reduced the proliferation ability of U251 
and U87 cells (Figure 3D-F). Human umbilical vein endothelia cells $\left(2 \times 10^{4}\right.$ cells / well $)$ were resuspended in the supernatant from sh-lncRNA-PVT1-U251 or sh-NC-U251 cells, and sh-lncRNA-PVT1-U87 or sh-NC-U87 cells. Then number of junctions and the total tubule length were chosen to assess the differences groups through Image $J$ software. Next, the supernatants from sh-lncRNA-PVT1-U251 and
sh-lncRNA-PVT1-U87 cells exhibited a strong negative effect on tubule formation of HUVECs, compared to control supernatants $(p<0.05)$ (Figure 3G-I). Transwell assays showed a similar result as tubule formation assays, sh-lncRNA-PVT1 significantly repressed the cell invation of glioma cancer cells (Figure 3J-L).

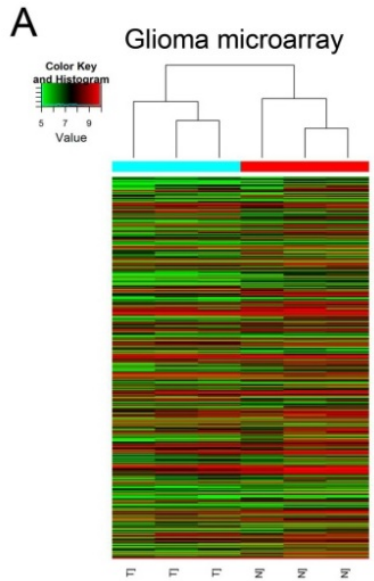

C

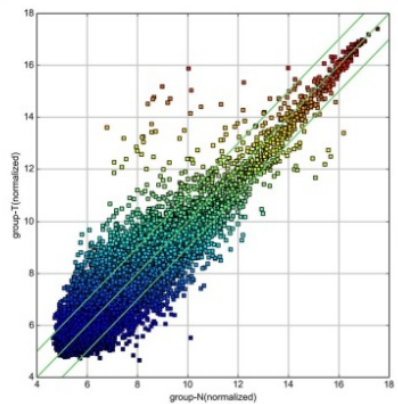

F
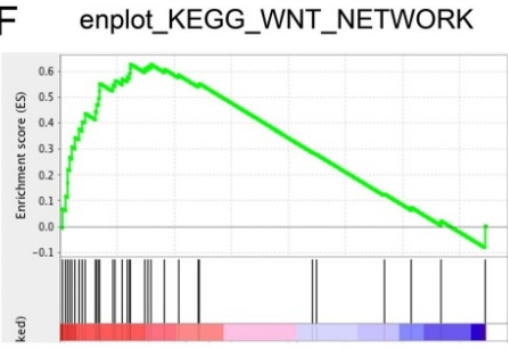

G enplot_KEGG_DNA_DAMAGE_REPAIR

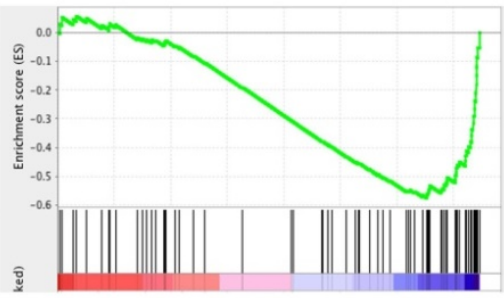

D
GSE29384
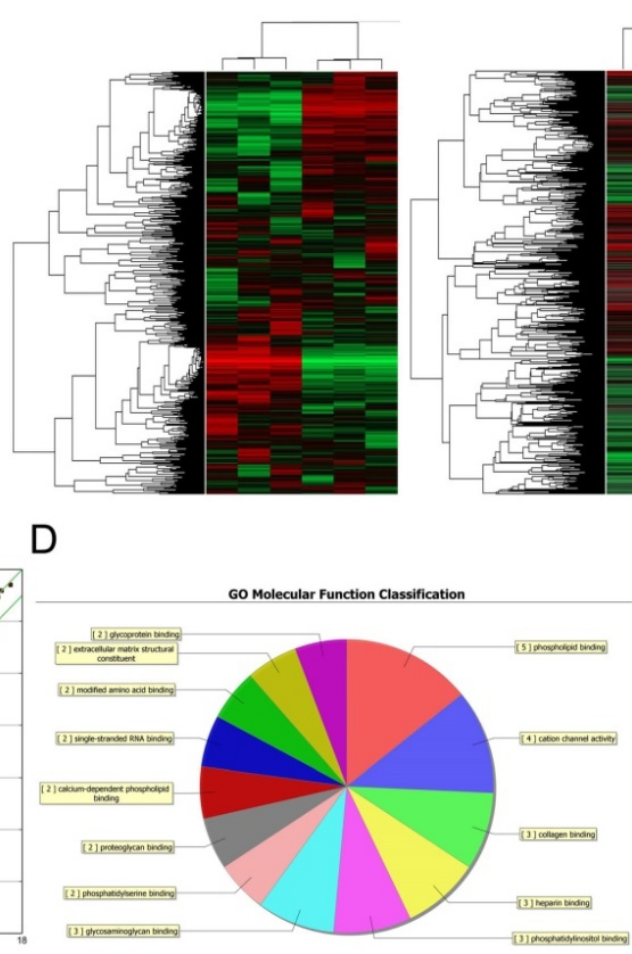

enplot_KEGG_TIGHT_JUNCTION

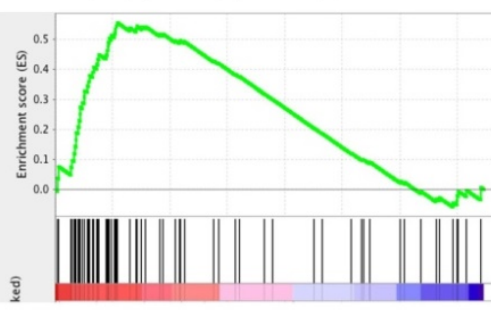

enplot_KEGG_T_CELL_RECEPTOR SIGNALING_PATHWAY

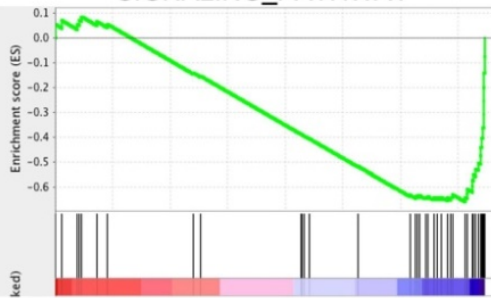

GSE7181

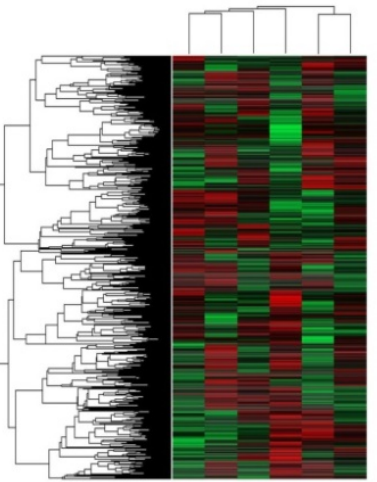

$E$

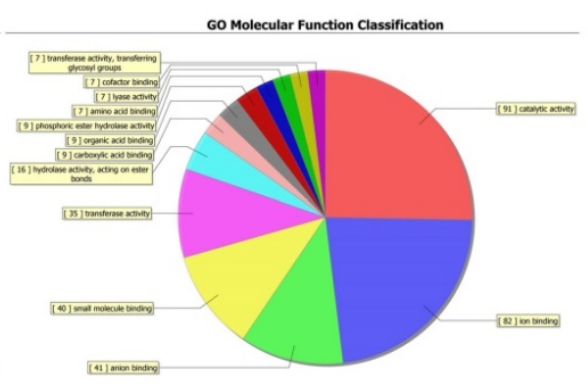

enplot_KEGG_CELL_ADHESION REABSORPTION

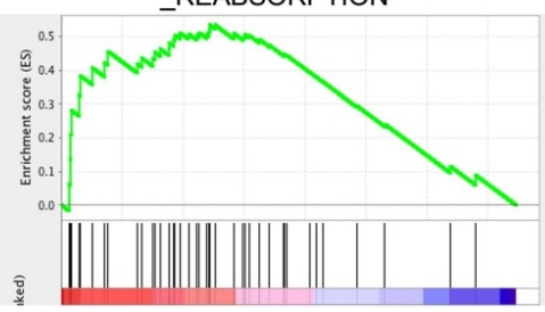

enplot_KEGG_OXIDATIVE_ STRESS_REACTION

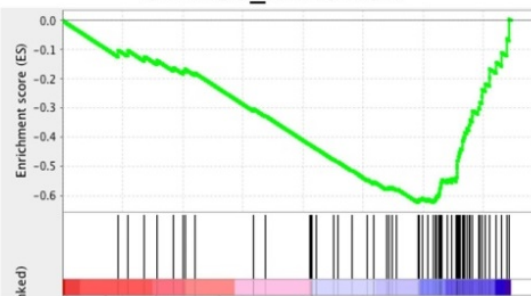

Figure 1. Dysregulated expression profiles of IncRNAs in glioma. (A) Aberrantly expression of IncRNAs was evaluated in 3 pairs of glioma and adjacent normal tissues though IncRNA expression microarray. (B) IncRNA-PVT1 expression in microarray datasets GSE29384, GSE7181 and GSE23806. (C) Scatter plot of gene expression in glioma compared with the control group (x-axis). Up-regulated IncRNAs are shown in red while down-regulated IncRNAs are shown in green. (D, E) GO biological process classification for differently expressed IncRNAs. The upper graph was related to the up-regulated IncRNAs (D) while the lower graph was related to the down-regulated IncRNAs (E). (F, G) GSEA revealed that proliferation and metastasis related biological functions were enriched in response to high IncRNA-PVT1 expression based on the microarray data set. 

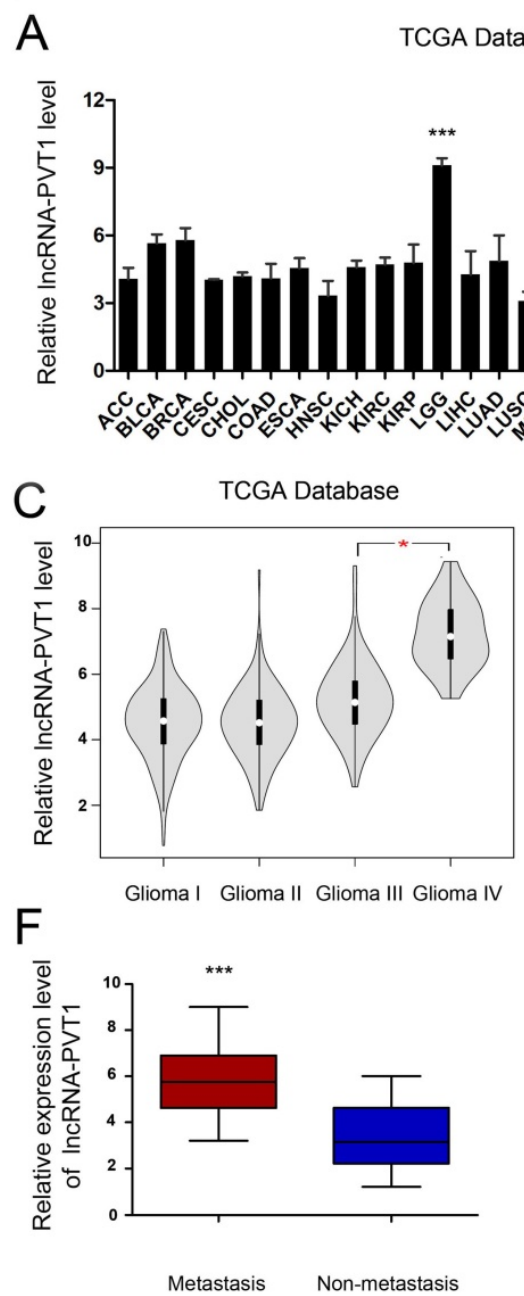

B

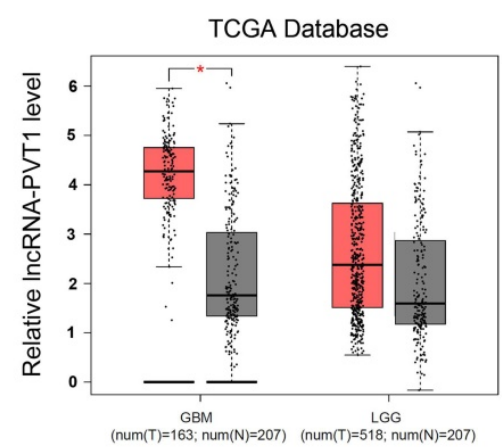

$E$

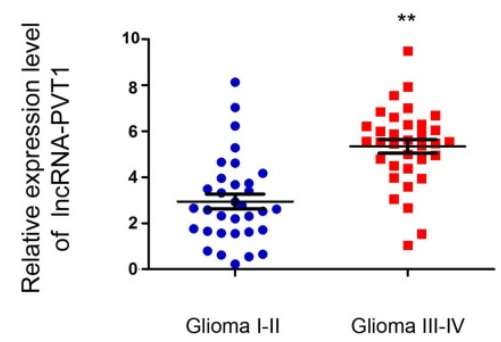

$\mathrm{H}$

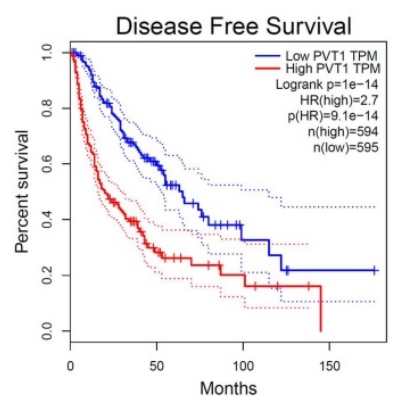

Figure 2. IncRNA-PVT1 was upregulated in glioma. (A, B) IncRNA-PVT1 expression in multiple solid tumors and normal tissues from TCGA Pan-Cancer dataset. (C) IncRNA-PVT1 expression in different TNM stage of glioma cases from TCGA dataset. (D) The IncRNA-PVT1 expression in glioma cases were determined by qRT-PCR assay. (E, F) High IncRNA-PVT1 expression was positively associated with advanced TNM stage and metastasis in patients with glioma. (G, H) The Kaplan-Meier curves introduced for survival analysis in glioma patients with high level and low level of IncRNA-PVT1. $* * p<0.01, * * * p<0.001$.

\section{Identification of IncRNA-PVT 1 target genes}

To further explore whether lncRNA-PVT1 serve as an endogenous RNAs (ceRNA) to exerts its function in glioma cells, we first examined lncRNA-PVT1 expression in the cytoplasmic and nuclear fractions of glioma cells. The results indicated that lncRNA-PVT1 was in the cytoplasm, which is the pre-condition of acting as a ceRNA (Figure 4A). We found that miR-1207-3p ranked top among all potential targets by bioinformatics analysis (miRcode, Starbase, and LncBase Predicted), the secondary structure and possible binding sites was shown in Figure 4B. The results illustrated that the expression of miR-1207-3p was pronouncedly increased in TCGA Pan-Cancer database (Figure 4D). The dual-luciferase reporter assay showed that the luciferase activity of HEK293 cells transfected with lncRNA-PVT1-Wt could be reduced by miR-1207-3p mimics (Figure 4E). Kaplan-Meier analysis in the TCGA database with glioma revealed that high lncRNA-PVT1 expression level was significantly correlated with a reduction in OS and DFS (Figure 4F), which is consistent with the important role of IncRNA-PVT1 in the pathogenesis and the prognosis of glioma in our study.

\section{IncRNA-PVT1 regulates the expression of HNFIB by competitive adsorption of miR-1207-3p}

Next, we explored the functions of miR-1207-3p in glioma. We further predicted the targeting sites between miR-1207-3p and HNF1B and found that miR-1207-3p shared complementary binding sites with HNF1B at 3'-UTR (Figure 5A). To verify the predicted results, dual-luciferase reporter assay showed that miR-1207-3p mimics significantly reduced luciferase activities of HNF1B-Wt group (Figure 5B). In addition, miR-1207-3p mimics significantly decreased HNF1B mRNA expression, while miR-1207-3p inhibitors increased HNF1B 
mRNA expression (Figure 5C). IHC results showed that both HNF1B protein expression was upregulated in glioma tissues and positively associated with advanced TNM stage (Figure 5D), which was further confirmed by TCGA database. Western blot results showed that overexpression of miR-1207-3p significantly down-regulated HNF1B compared with the control group, suggesting that HNF1B expression is inversely regulated by miR-1207-3p in glioma (Figure 5E, F). Those data indicated that HNF1B and foxm1 might play critical roles in glioma progression.
A

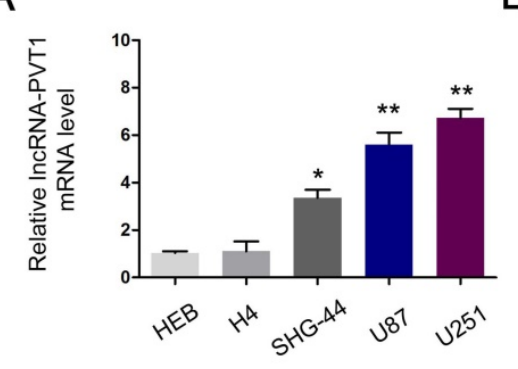

B

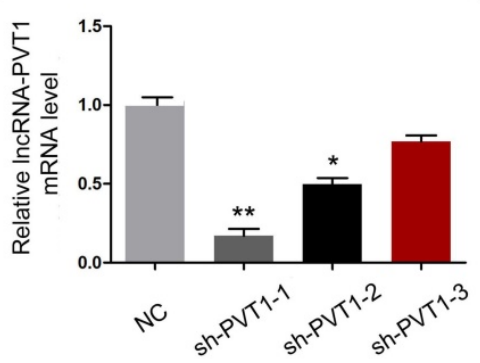

C

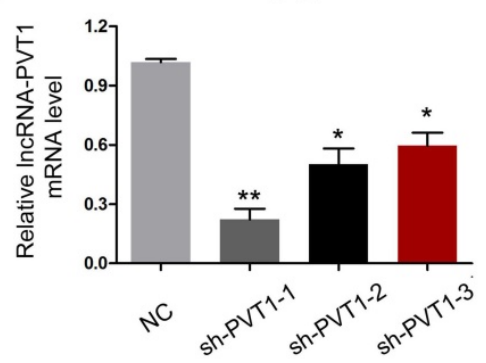

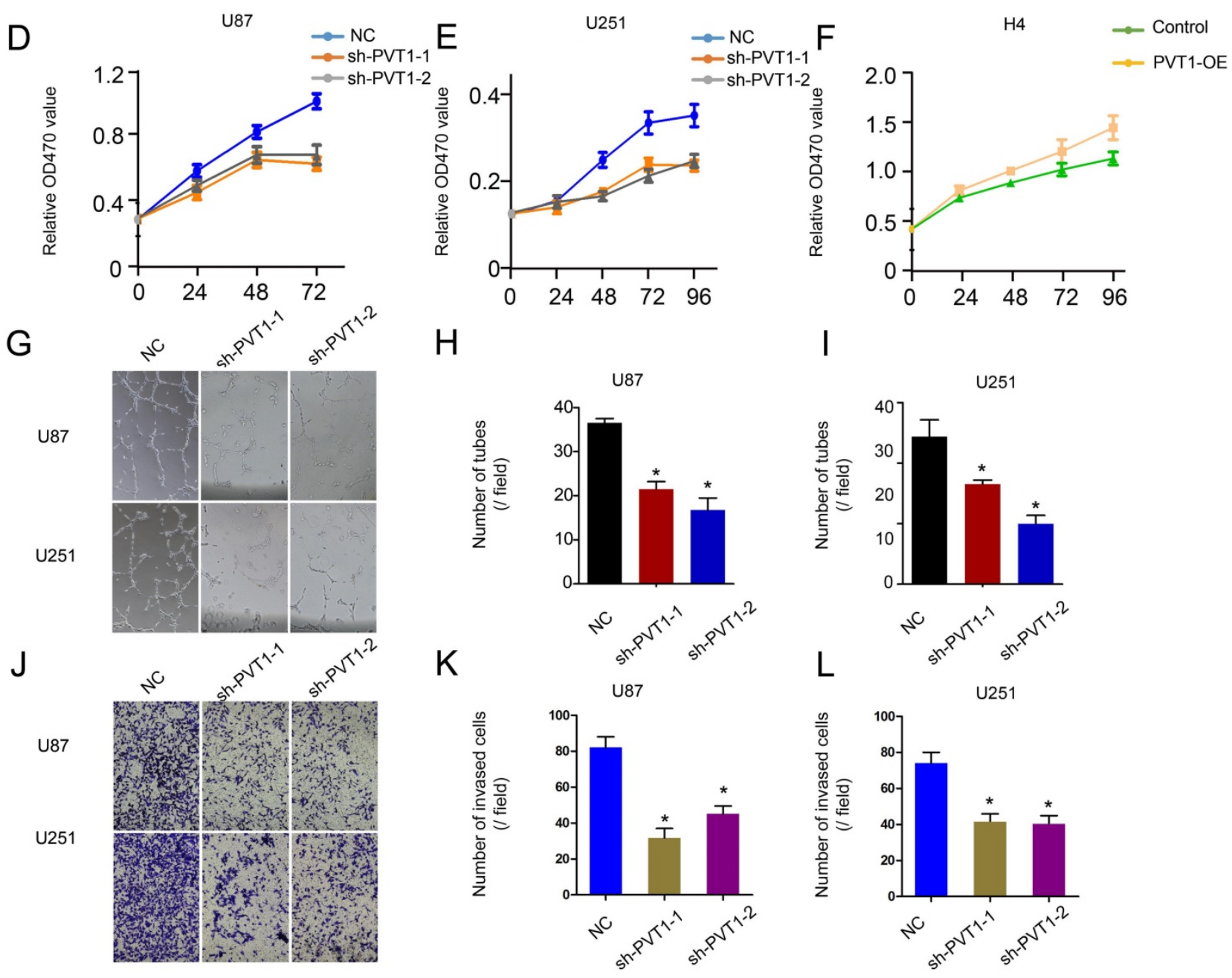

Figure 3. IncRNA-PVT1 promotes glioma the development of pro-metastatic phenotype in vitro. (A) IncRNA-PVT1 expression in glioma cell lines (H4, SHG-44, U87 and U251) and HEB was detected by qRT-PCR assay. (B, C) Knockdown of IncRNA-PVT1 by shRNA in U87 and U251 cells. (D-F) MTT assay was performed to assess the function of IncRNA-PVT1 in glioma cells proliferation. (G-I) The supernatants from sh-IncRNA-PVT1 cells exhibited a strong negative effect on tubule formation of HUVECs, compared to control supernatants in U87 and U251 cells. (J-L) Knockdown of IncRNA-PVT1 in U251 and U87 cells significantly inhibited cell invasion. We selected the 1\# and 2\# sh-plasmids with high interference efficiency for testing. ${ }^{*} p<0.05$. 
A

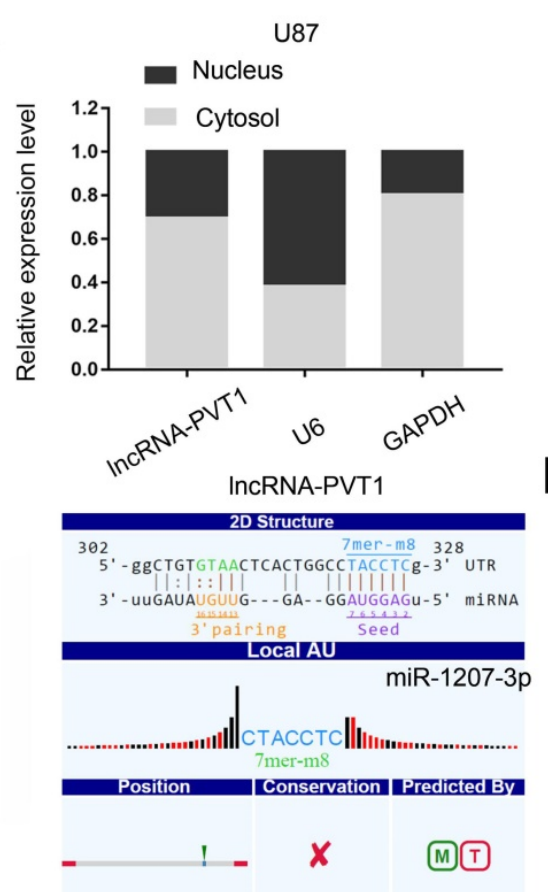

$E$

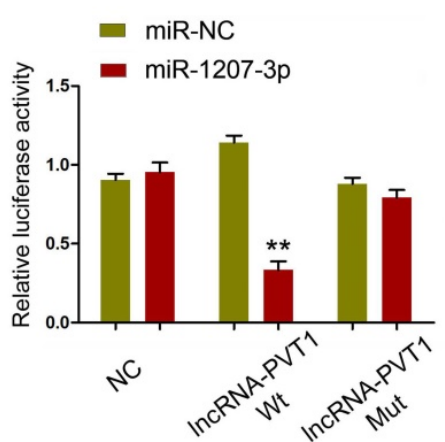

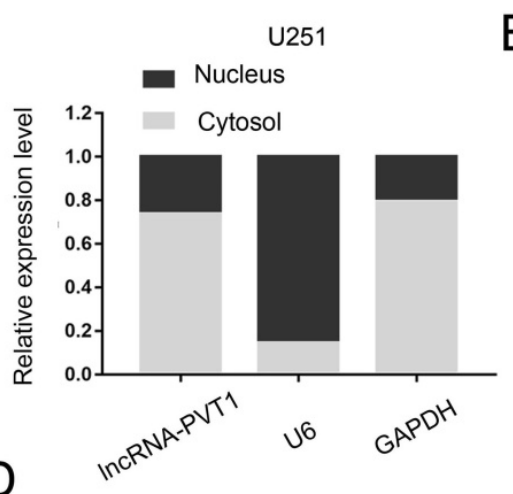

B

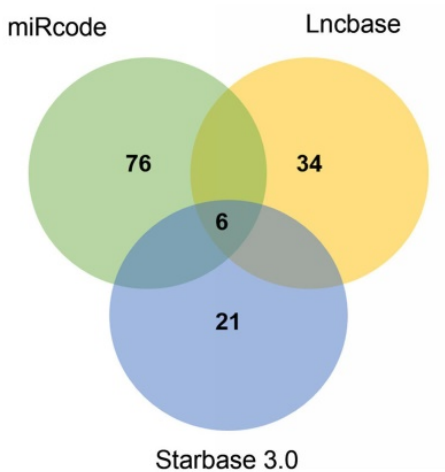

TCGA Database Pan-Cancer

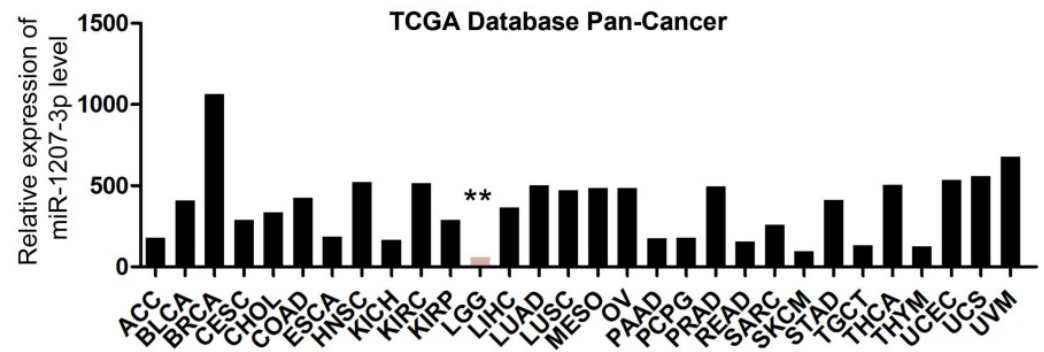

$\mathrm{F}$

Overall Survival from TCGA Database Disease-free Survival from TCGA Database miR-1207-3p

miR-1207-3p
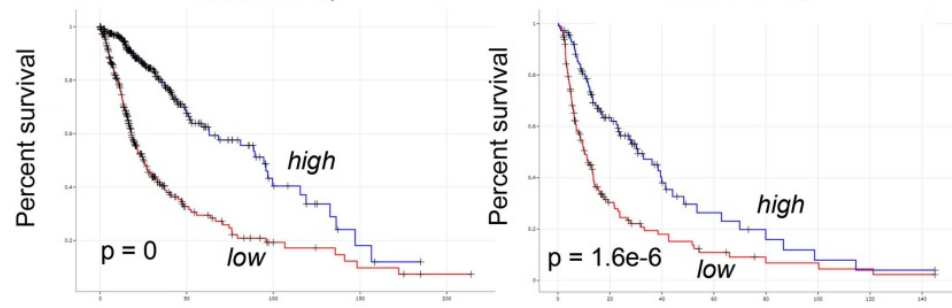

Figure 4. IncRNA-PVT1 acted as a sponge for miR-1207-3p. (A) IncRNA-PVT1 was located in the cytoplasm of U87 and U251 cells. (B) Venn diagrams showed the results of potential targets of IncRNA-PVT1 (LncBase Predicted, Starbase 3.0 and miRcode databases). (C) Bioinformatics analysis shows that IncRNA-PVT1 contains one conserved target binding site of miR-1207-3p, the predicted complementary binding sites between IncRNA-PVT1 and miR-1207-3p. (D) miR-1207-3p expression in multiple solid tumors and normal tissues from TCGA Pan-Cancer dataset. (E) Dual-luciferase assay showed miR-1207-3p mimics reduced the luciferase activity of IncRNA-PVT1-Wt. (F) Low miR-1207-3p expression was associated with poor OS and DFS in patients with glioma. $* * p<0.001$.

\section{IncRNA-PVT1/miR-1207-3p/HNF IB axis promoted glioma progression}

To determine whether the effects of lncRNAPVT1 in glioma cells metastasis were dependent on miR-1207-3p/HNF1B axis, we further tested the roles of lncRNA-PVT1/miR-1207-3p/HNF1B axis in glioma progression. Furthermore, we examined the effects of lncRNA-PVT1 in xenograft mouse model. Results revealed that lncRNA-PVT1 inhibition also significantly reduced tumor growth in vivo (Figure 6A-C). Overall, these results indicated that lncRNA-PVT1 silencing significantly reduced the proliferation and invasion ability of glioma cells in vitro and reduced tumor growth in vivo. EMT processes play critical roles in tumor metastasis. In the present study, western blot showed that lncRNA-PVT1 inhibition significantly reduced EMT related gene expression (Vimentin and $\mathrm{N}$-cadherin) and MAPK pathway related gene expression (foxm1, VEGFA and PKP4) expression. And the effects of lncRNA-PVT1 inhibition on MAPK signaling pathway and EMT processes could be restored by miR-1207-3p shRNAs (Figure 6F). Thus, our data suggested that IncRNA-PVT1 promoted the proliferation and metastasis of glioma by regulating miR-1207-3p/HNF1B/MAPK pathway (Figure 6D-E). 
A

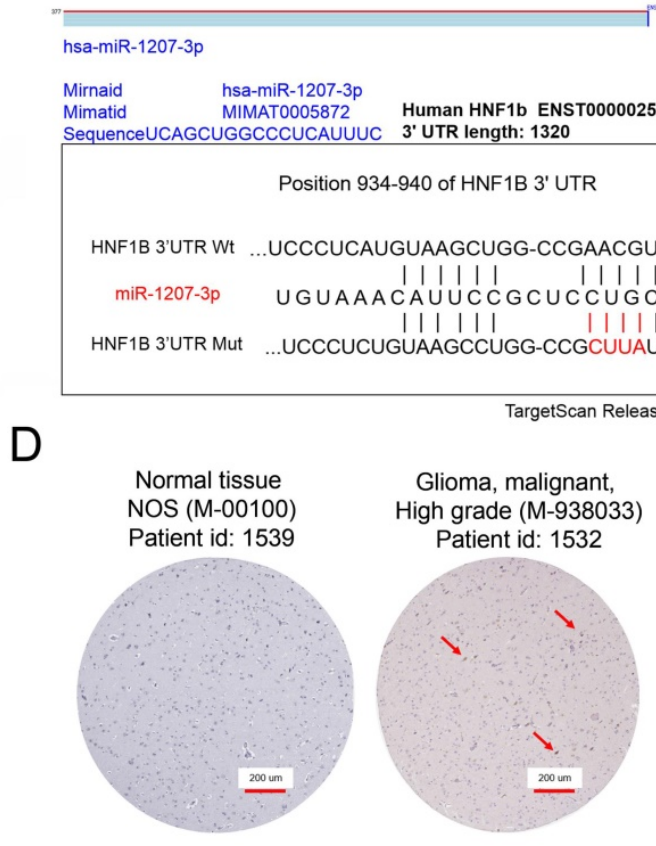

B

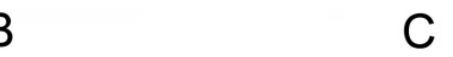

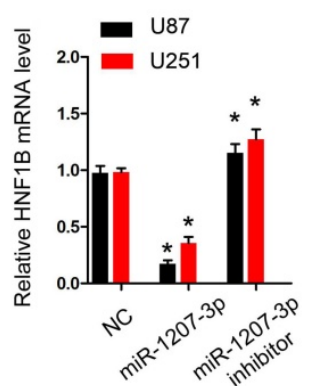

$\mathrm{F}$
E

U87
U251
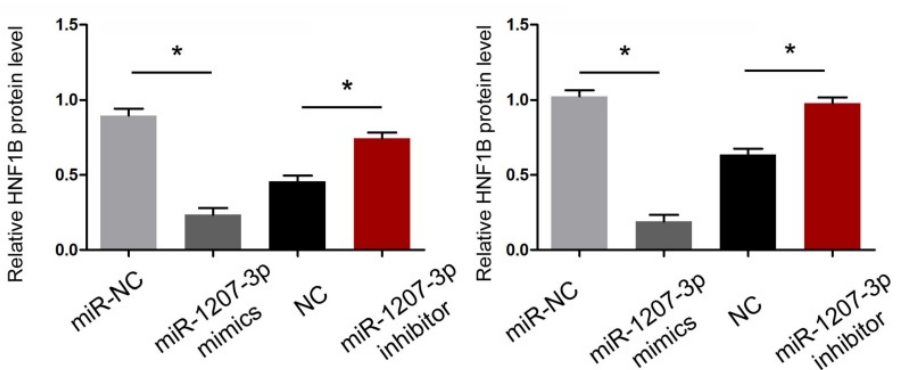

Figure 5. HNF1B is the direct target gene of miR-1207-3p and associated with poor prognosis of glioma. (A) The predicted complementary binding sites between miR-1207-3p and HNF1B. (B) Dual-luciferase assay showed miR-1207-3p mimics reduced the luciferase activity of HNF1B-Wt. (C) miR-1207-3p mimics reduced HNF1B expression in glioma cells, while miR-1207-3p inhibitors increased HNF1B mRNA expression. (D) IHC assays showed HNF1B expression was upregulated and associated advanced TNM stage in patients with glioma. (E, F) Western blot results showed overexpression of miR-489 significantly down-regulated HNF1B in U87 and U251 cells. *p<0.05.

A

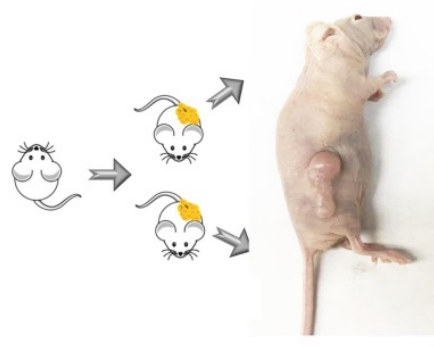

sh-IncRNA-PVT1

D

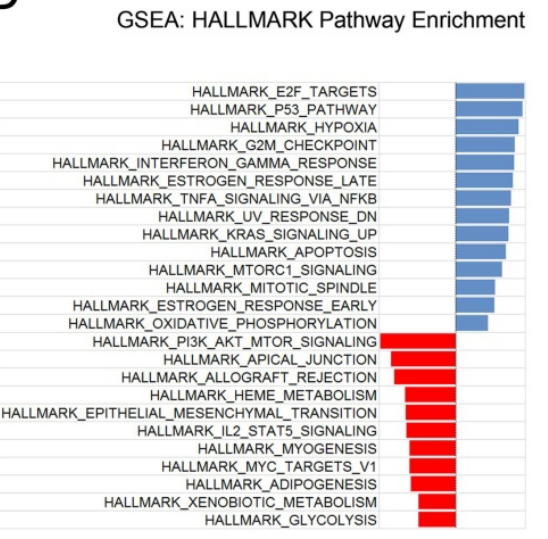

B

sh-IncRNA-NC Tumor xenograft implantation in nude mice

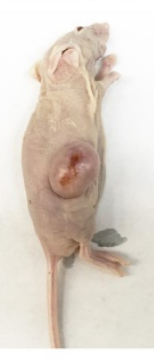

E

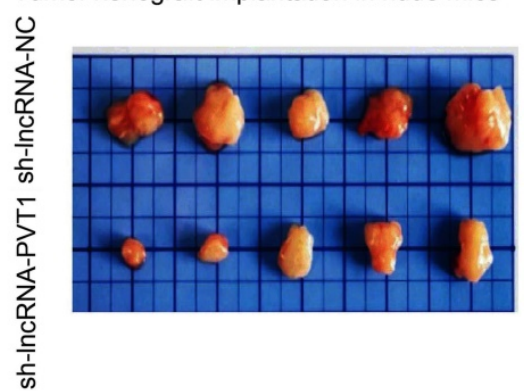

GSEA: KEGG Pathway Enrichment

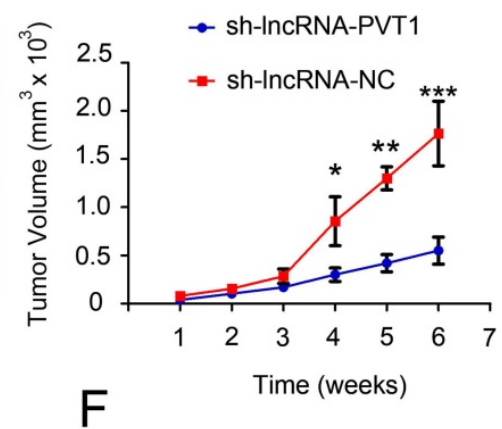

$\mathrm{F}$

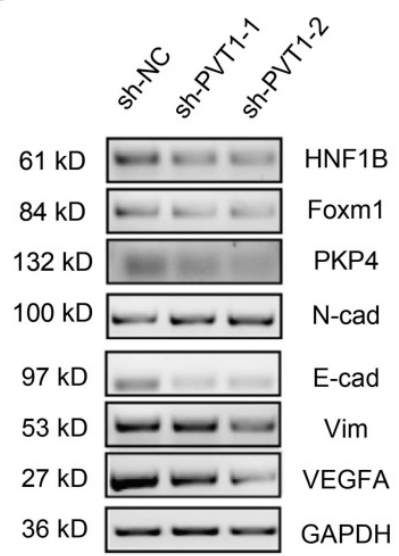

Figure 6. IncRNA-PVT1 inhibition reduced tumor growth in vivo, and IncRNA-PVT1/miR-1207-3p/HNF1B axis promoted glioma metastasis. (A) Representative images of tumors in xenograft mouse model. (B)The relative weights of xenograft tumors. (C) The growth curves of xenograft tumors. (D, E) HALLMARK and KEGG Pathway Enrichment in glioma from microarry datasets. The datasets were analyzed using the Hallmark gene signature collection. Hallmark_EMT was identified as the pathway with the top two-highest association. (F) The expressions of HNF1B, EMT-related makers and VEGF-related molecules were determined using western blot. Proteins were isolated from cells transfected as indicated. $* p<0.05, * * p<0.01, * * * p<0.001$ 


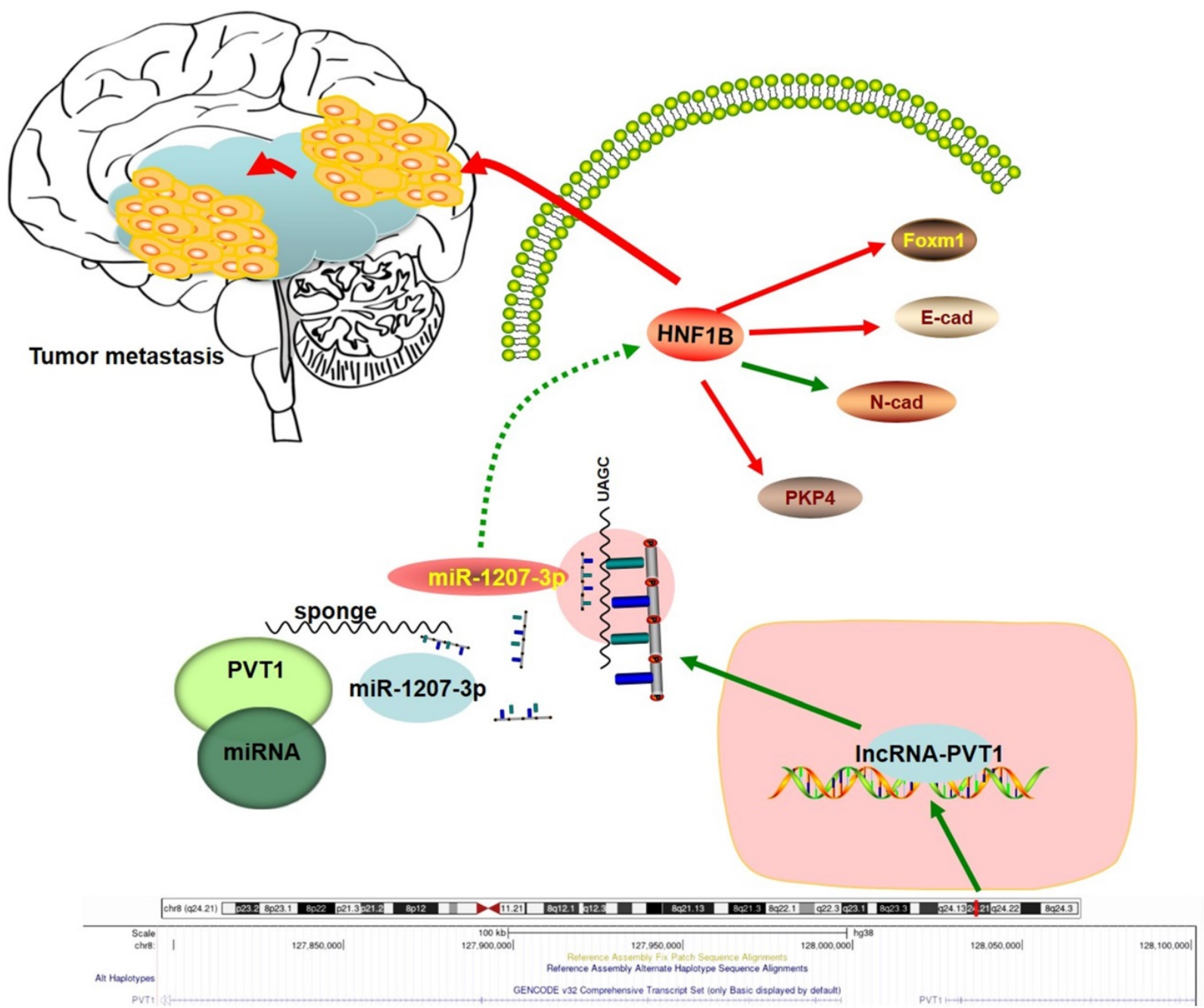

Figure 7. Schema diagram of IncRNA-PVT1-miR-1207-3p/HNF1B axis in human glioma.

\section{Discussion}

At present, only a few functional mechanisms of lncRNAs have been well characterized, and some recent insights into IncRNAs identified in other diseases and cancers, such as molecules encoding tumor-related micropeptides, changes in the activities or conformation of proteins and the regulation of tumor microenvironment homeostasis, have not yet been clearly demonstrated in glioma pathogenesis [33]. The majority of studies of glioma-associated lncRNAs are typically focused on ceRNA regulatory networks [34]. Thus, there is an urgent need to elucidate the detailed mechanisms of the roles of lncRNAs in glioma.

Glioma displays complex etiology involving multiple risk factors, such as environmental exposure, inherited genetic alterations, and multiple epigenetic modifications based on global molecular biomarkers, including mRNAs and lncRNAs [35, 36]. Although surgical resection is the best chance for a possible cure, the prognosis of glioma remains poor [37]. Therefore, it is highly desirable to identify and characterize genes that play important roles in glioma pathogenesis. Among the critical molecules involved in the glioma progression, lncRNAs gradually become an attractive therapeutic target for cancers [38]. For example, Yang et al. found that lncRNA-BANCR promoted non-small-cell lung cancer cells growth and metastasis via affecting EMT processes [39]. Fu et al. found that IncRNA-HOTTIP modulated cancer stem cell properties through regulating HMGA1 in human gastric cancer [40]. Jin et al. suggested that lncRNA-HOTTIP enhances osteogenic differentiation via interaction with WDR5 and up-regulation of $\beta$-catenin gene expression, therefore activating Wnt/ $\beta$-catenin signalling pathway [41]. Additionally, whether lncRNA expression in glioma is dysregulated and abnormal remains elusive, and the underlying mechanisms are still unknown.

LncRNA-PVT1 is reported to be involved in tumorigenesis and progression of many malignancies, 
including cervical cancer, nonsmall cell lung cancer, pancreatic cancer, and so forth [42]. In the recent years, some emerging evidence has suggested that silencing PVT1 could inhibit malignant biological behaviors of glioma cells [43]. For instance, Xue et al. unraveled that knockdown of PVT1 weakened the malignant behaviors of glioma cells via the inhibition of cell motility and invasiveness [44]. Wang et al. also substantiated that downregulation of PVT1 expression resulted in decreased cell viability and mobility and increased apoptosis [45]. In addition, lncRNAs can act as competing endogenous RNA sponges for miRNAs to regulate the degradation of miRNA targets, thereby influencing post-transcriptional regulation. In our study, we revealed that IncRNAPVT1 was obviously increased in glioma tissues as well as in glioma cell lines. High lncRNA-PVT1 expression was positively associated with advanced TNM stage, metastasis, and poor prognosis in patients with glioma. In addition, loss-of-function assays demonstrated that lncRNA-PVT1 inhibition suppressed the proliferation and invasion in glioma cells in vitro and reduced tumor growth in vivo. Then the regulatory relationship between lncRNA-PVT1 and miR-1207-3p was validated through bioinformatics analysis, luciferase reporter assay as well as FISH assay. The present study further confirmed that transcriptional activation of lncRNA-PVT1 contributed to oncogenesis, whereas knockdown of lncRNA-PVT1 impaired oncogenic function of HNF1B in glioma cells. These data suggested that lncRNA-PVT1 could play critical roles in glioma tumorigenesis.

HNF1B could play critical functions in proliferation, metastasis, apoptosis, and linked to EMT pathway in several cancers [46-49]. In our study, by bioinformatic prediction and experimental verification, we showed that HNF1B could act as a direct target of miR-1207-3p in glioma. Furthermore, we showed that the anti-metastatic roles of lncRNA-PVT1 inhibition on glioma cells invasion could be abolished by co-transfected with miR1207-3p inhibitors (or pcDNA3.1_HNF1B plasmid). In addition, western blot revealed that IncRNA-PVT1 inhibition significantly reduced the expression of EMT pathway related genes. And the effects of lncRNA-PVT1 inhibition on EMT pathway could be restored by co-transfected with miR-1207-3p inhibitors. Therefore, these findings suggested that IncRNA-PVT1 promoted the proliferation and invasion in glioma via regulating miR-1207-3pmediated HNF1B/EMT pathway.

In summary, our study identified the interaction between lncRNA-PVT1, miR-1207-3p and HNF1B in glioma (Figure 7). In the present study, miR-1207-3p was predicted as the target of lncRNA-PVT1.
Dual-luciferase reporter assay confirmed the correlation between lncRNA-PVT1 and miR-1207-3p. Furthermore, transwell invasion assay further revealed that lncRNA-PVT1 promoted glioma cells invasion by downregulating miR-1207-3p expression. Overall, we suggested that lncRNA-PVT1 could act as a sponge for miR-1207-3p in glioma. And the lncRNA-PVT1/miR-1207-3p/HNF1B/MAPK axis might provide a new potential therapeutic target for treatment of glioma.

\section{Acknowledgments}

We are appreciating to every author for their assistance with the experiments and constructive comments on the manuscript.

\section{Ethics approval and consent to participate}

Ethical approval was given by the Ethics Committee of Fudan University.

\section{Availability of data and materials}

The dataset supporting the conclusions of this article is included within the article.

\section{Author Contributions}

Yongyan Bi, Jie Ji, Youxin Zhou conceived and designed the study. Yongyan Bi performed all the in vitro experiments. Yongyan Bi, Jie Ji, Youxin Zhou provided support with experimental techniques. Yongyan Bi, Jie Ji, Youxin Zhou collected clinical data. Yongyan $\mathrm{Bi}$ analyzed the data and prepared the manuscript. Yongyan Bi, Jie Ji, Youxin Zhou reviewed and revised the manuscript. Yongyan Bi, Jie Ji, Youxin Zhou provided material support and supervised all experiments. All authors read and approved the final manuscript.

\section{Funding}

This study was supported by Minhang District Natural Science Foundation Project (No. 2020MHZ084) and Minhang district High-level Specialized Backbone Doctor Training plan.

\section{Competing Interests}

The authors have declared that no competing interest exists.

\section{References}

1. Reifenberger $G$, Wirsching $H G$, Knobbe-Thomsen $C B$, Weller $M$. Advances in the molecular genetics of gliomas - implications for classification and therapy. Nat Rev Clin Oncol. 2017;14(7):434-452.

2. Bi J, Chowdhry S, Wu S, Zhang W, Masui K, Mischel PS. Altered cellular metabolism in gliomas - an emerging landscape of actionable co-dependency targets. Nat Rev Cancer. 2020 Jan;20(1):57-70.

3. Chen R, Smith-Cohn M, Cohen AL, Colman H. Glioma Subclassifications and Their Clinical Significance. Neurotherapeutics. 2017 Apr;14(2):284-297.

4. Gusyatiner O, Hegi ME. Glioma epigenetics: From subclassification to novel treatment options. Semin Cancer Biol. 2018 Aug;51:50-58. 
5. Hervey-Jumper SL, Berger MS. Insular glioma surgery: an evolution of thought and practice. J Neurosurg. 2019 Jan 1;130(1):9-16.

6. Puvvula PK. LncRNAs Regulatory Networks in Cellular Senescence. Int J Mol Sci. 2019 May 28;20(11):2615.

7. Grote P, Boon RA. LncRNAs Coming of Age. Circ Res. 2018 Aug 17;123(5):535-537.

8. Wu T, Du Y. LncRNAs: From Basic Research to Medical Application. Int J Biol Sci. 2017 Feb 23;13(3):295-307.

9. Meng Y, Qiu S, Sun L, Zuo J. Knockdown of exosome-mediated lnc-PVT1 alleviates lipopolysaccharide-induced osteoarthritis progression by mediating the HMGB1/TLR4/NF-kB pathway via miR-93-5p. Mol Med Rep. 2020 Dec;22(6):5313-5325.

10. Qiu C, Li S, Sun D, Yang S. IncRNA PVT1 accelerates progression of non-small cell lung cancer via targeting miRNA-526b/EZH2 regulatory loop. Oncol Lett. 2020 Feb;19(2):1267-1272.

11. Chang QQ, Chen CY, Chen Z, Chang S. LncRNA PVT1 promotes proliferation and invasion through enhancing Smad3 expression by sponging miR-140-5p in cervical cancer. Radiol Oncol. 2019 Oct 18;53(4):443-452.

12. Pan X, Cheng R, Zhu X, Cai F, Zheng G, Li J, Gao C. Prognostic Significance and Diagnostic Value of Overexpressed lncRNA PVT1 in Colorectal Cancer. Clin Lab. 2019 Dec 1;65(12).

13. Zhang XW, Bu P, Liu L, Zhang XZ, Li J. Overexpression of long non-coding RNA PVT1 in gastric cancer cells promotes the development of multidrug resistance. Biochem Biophys Res Commun. 2015 Jul 3;462(3):227-32.

14. Lu D, Luo P, Wang Q, Ye Y, Wang B. lncRNA PVT1 in cancer: A review and meta-analysis. Clin Chim Acta. 2017 Nov;474:1-7.

15. Tang J, Li Y, Sang Y, Yu B, Lv D, Zhang W, Feng H. LncRNA PVT1 regulates triple-negative breast cancer through KLF5/beta-catenin signaling. Oncogene. 2018 Aug;37(34):4723-4734.

16. Yoshida K, Toden S, Ravindranathan P, Han H, Goel A. Curcumin sensitizes pancreatic cancer cells to gemcitabine by attenuating PRC2 subunit EZH2, and the lncRNA PVT1 expression. Carcinogenesis. 2017 Oct 1;38(10):1036-1046

17. Xu MD, Wang Y, Weng W, Wei P, Qi P, Zhang Q, Tan C, Ni SJ, Dong L, Yang Y, Lin W, Xu Q, Huang D, Huang Z, Ma Y, Zhang W, Sheng W, Du $X$. A Positive Feedback Loop of lncRNA-PVT1 and FOXM1 Facilitates Gastric Cancer Growth and Invasion. Clin Cancer Res. 2017 Apr 15;23(8):2071-2080.

18. Wang Y, Chen W, Lian J, Zhang H, Yu B, Zhang M, Wei F, Wu J, Jiang J, Jia Y, Mo F, Zhang S, Liang X, Mou X, Tang J. The lncRNA PVT1 regulates nasopharyngeal carcinoma cell proliferation via activating the KAT2A acetyltransferase and stabilizing HIF-1a. Cell Death Differ. 2020 Feb;27(2):695-710.

19. Han Y, Li X, He F, Yan J, Ma C, Zheng X, Zhang J, Zhang D, Meng C, Zhang Z, Ji X. Knockdown of lncRNA PVT1 Inhibits Glioma Progression by Regulating miR-424 Expression. Oncol Res. 2019 Jun 21;27(6):681-690.

20. Zhang Y, Yang G, Luo Y. Long non-coding RNA PVT1 promotes glioma cell proliferation and invasion by targeting miR-200a. Exp Ther Med. 2019 Feb;17(2):1337-1345.

21. Li W, Liu M, Feng Y, Xu YF, Huang YF, Che JP, Wang GC, Yao XD, Zheng JH. Downregulated miR-646 in clear cell renal carcinoma correlated with tumour metastasis by targeting the nin one binding protein (NOB1). Br J Cancer. 2014 Sep 9;111(6):1188-200.

22. Li W, Yang FQ, Sun CM, Huang JH, Zhang HM, Li X, Wang GC, Zhang N, Che JP, Zhang WT, Yan Y, Yao XD, Peng B, Zheng JH, Liu M. circPRRC2A promotes angiogenesis and metastasis through epithelial-mesenchymal transition and upregulates TRPM3 in renal cell carcinoma. Theranostics. 2020 Mar 15;10(10):4395-4409.

23. Zhang H, Li W, Gu W, Yan Y, Yao X, Zheng J. MALAT1 accelerates the development and progression of renal cell carcinoma by decreasing the expression of miR-203 and promoting the expression of BIRC5. Cell Prolif. 2019 Sep;52(5):e12640.

24. Zhao JJ, Lin J, Zhu D, Wang X, Brooks D, Chen M, Chu ZB, Takada K, Ciccarelli B, Admin S, Tao J, Tai YT, Treon S, Pinkus G, Kuo WP, Hideshima T, Bouxsein M, Munshi N, Anderson $K$, Carrasco R. miR-30-5p functions as a tumor suppressor and novel therapeutic tool by targeting the oncogenic Wnt/ $\beta$-catenin/BCL9 pathway. Cancer Res. 2014 Mar 15;74(6):1801-13.

25. Han X, Zhen S, Ye Z, Lu J, Wang L, Li P, Li J, Zheng X, Li H, Chen W, Li $X$, Zhao L. A Feedback Loop Between miR-30a/c-5p and DNMT1 Mediates Cisplatin Resistance in Ovarian Cancer Cells. Cell Physiol Biochem. 2017;41(3):973-986.

26. Cao JM, Li GZ, Han M, Xu HL, Huang KM. MiR-30c-5p suppresses migration, invasion and epithelial to mesenchymal transition of gastric cancer via targeting MTA1. Biomed Pharmacother. 2017 Sep;93:554-560.
27. Das DK, Osborne JR, Lin HY, Park JY, Ogunwobi OO. miR-1207-3p Is a Novel Prognostic Biomarker of Prostate Cancer. Transl Oncol. 2016 Jun;9(3):236-41.

28. Anauate AC, Leal MF, Wisnieski F, Santos LC, Gigek CO, Chen ES, Calcagno DQ, Assumpção PP, Demachki S, Arasaki $\mathrm{CH}$, Artigiani R, Rasmussen LT, Payão SM, Burbano RR, C Smith MA. Analysis of 8q24.21 miRNA cluster expression and copy number variation in gastric cancer. Future Med Chem. 2019 May;11(9):947-958.

29. You L, Wang H, Yang G, Zhao F, Zhang J, Liu Z, Zhang T, Liang Z, Liu C, Zhao Y. Gemcitabine exhibits a suppressive effect on pancreatic cancer cell growth by regulating processing of PVT1 to miR1207. Mol Oncol. 2018 Dec;12(12):2147-2164

30. Barsotti AM, Beckerman R, Laptenko O, Huppi K, Caplen NJ, Prives C. p53-Dependent induction of PVT1 and miR-1204. J Biol Chem. 2012 Jan 20;287(4):2509-19.

31. Zhang Y, Li L, Liang P, Zhai X, Li Y, Zhou Y. Differential Expression of microRNAs in Medulloblastoma and the Potential Functional Consequences. Turk Neurosurg. 2018;28(2):179-185.

32. Rodríguez A, Rodríguez M, Córdoba JJ, Andrade MJ. Design of primers and probes for quantitative real-time PCR methods. Methods Mol Biol. 2015;1275:31-56.

33. Liu X, Zheng J, Xue Y, Yu H, Gong W, Wang P, Li Z, Liu Y. PIWIL3/OIP5-AS1/miR-367-3p/CEBPA feedback loop regulates the biological behavior of glioma cells. Theranostics. 2018 Jan 1;8(4):1084-1105.

34. Liu ZZ, Tian YF, Wu H, Ouyang SY, Kuang WL. LncRNA H19 promotes glioma angiogenesis through miR-138/HIF-1a/VEGF axis. Neoplasma. 2020 Jan;67(1):111-118.

35. Zhu J, Gu W, Yu C. MATN1-AS1 promotes glioma progression by functioning as ceRNA of miR-200b/c/429 to regulate CHD1 expression. Cell Prolif. 2020 Jan;53(1):e12700.

36. Liang Q, Li X, Guan G, Xu X, Chen C, Cheng P, Cheng W, Wu A. Long non-coding RNA, HOTAIRM1, promotes glioma malignancy by forming a ceRNA network. Aging (Albany NY). 2019 Sep 2;11(17):6805-6838.

37. Kraus TF, Greiner A, Guibourt V, Lisec K, Kretzschmar HA. Identification of Stably Expressed lncRNAs as Valid Endogenous Controls for Profiling of Human Glioma. J Cancer. 2015 Jan 1;6(2):111-9.

38. Shen J, Xiong J, Shao X, Cheng H, Fang X, Sun Y, Di G, Mao J, Jiang X. Knockdown of the long noncoding RNA XIST suppresses glioma progression by upregulating miR-204-5p. J Cancer. 2020 May 18:11(15):4550-4559.

39. Yang L, Liu G. IncRNA BANCR suppresses cell viability and invasion and promotes apoptosis in non-small-cell lung cancer cells in vitro and in vivo. Cancer Manag Res. 2019 Apr 26;11:3565-3574

40. Wang J, Lv B, Su Y, Wang X, Bu J, Yao L. Exosome-Mediated Transfer of lncRNA HOTTIP Promotes Cisplatin Resistance in Gastric Cancer Cells by Regulating HMGA1/miR-218 Axis. Onco Targets Ther. 2019 Dec 20;12:11325-11338.

41. Liu R, Li Z, Song E, Hu P, Yang Q, Hu Y, Liu H, Jin A. LncRNA HOTTIP enhances human osteogenic BMSCs differentiation via interaction with WDR5 and activation of Wnt/ $\beta$-catenin signalling pathway. Biochem Biophys Res Commun. 2020 Apr 16;524(4):1037-1043.

42. Liu G, Liu S, Xing G, Wang F. IncRNA PVT1/MicroRNA-17-5p/PTEN Axis Regulates Secretion of E2 and P4, Proliferation, and Apoptosis of Ovarian Granulosa Cells in PCOS. Mol Ther Nucleic Acids. 2020 Jun 5;20:205-216

43. Shao Y, Chen HT, Ma QR, Zhang YW, He YQ, Liu J. Long non-coding RNA PVT1 regulates glioma proliferation, invasion, and aerobic glycolysis via miR-140-5p. Eur Rev Med Pharmacol Sci. 2020 Jan;24(1):274-283.

44. Xue W, Chen J, Liu X, Gong W, Zheng J, Guo X, Liu Y, Liu L, Ma J, Wang $P, L i Z, X u e$ Y. PVT1 regulates the malignant behaviors of human glioma cells by targeting miR-190a-5p and miR-488-3p. Biochim Biophys Acta Mol Basis Dis. 2018 May;1864(5 Pt A):1783-1794.

45. Zhan J, Hu P, Wang Y. lncRNA PVT1 aggravates doxorubicin-induced cardiomyocyte apoptosis by targeting the miR-187-3p/AGO1 axis. Mol Cell Probes. 2020 Feb;49:101490.

46. Lu W, Sun J, Zhou H, Wang F, Zhao C, Li K, Fan C, Ding G, Wang J. HNF1B inhibits cell proliferation via repression of SMAD6 expression in prostate cancer. J Cell Mol Med. 2020 Dec;24(24):14539-14548.

47. Bubancova I, Kovarikova H, Laco J, Ruszova E, Dvorak O, Palicka V, Chmelarova M. Next-Generation Sequencing Approach in Methylation Analysis of HNF1B and GATA4 Genes: Searching for Biomarkers in Ovarian Cancer. Int J Mol Sci. 2017 Feb 22;18(2):474.

48. Harries LW, Perry JR, McCullagh P, Crundwell M. Alterations in LMTK2, MSMB and HNF1B gene expression are associated with the development of prostate cancer. BMC Cancer. 2010 Jun 22;10:315.

49. Lerner J, Bagattin A, Verdeguer F, Makinistoglu MP, Garbay S, Felix T, Heidet L, Pontoglio M. Human mutations affect the 
epigenetic/bookmarking function of HNF1B. Nucleic Acids Res. 2016 Sep 30;44(17):8097-111. 